\title{
Aspectos Econômicos em Gerenciamento de Recursos de Rádio
}

\author{
Raimundo A. de Oliveira Neto* e Francisco Rodrigo P. Cavalcanti
}

\begin{abstract}
Resumo-Neste artigo nós abordamos o papel e a importância de aspectos econômicos essenciais para o sucesso do Gerenciamento de Recursos de Rádio em redes de telefonia celular do ponto de vista financeiro. Entre os principais aspectos econômicos que podem influenciar a operação da rede, podemos destacar os modelos de tarifação, o comportamento do consumidor de serviços de telecomunicação sem fio e a concorrência entre operadoras de telefonia celular. Para evidenciar as vantagens de um Gerenciamento de Recursos de Rádio baseado em aspectos econômicos, nós realizamos um estudo de caso através de simulação computacional.
\end{abstract}

Palavras-Chave-Gerenciamento de Recursos de Rádio (RRM), Microeconomia, Comportamento do Consumidor.

Abstract-In this paper we deal with the role and the importance of economic aspects essential to the success of the Radio Resource Management in cellular networks from a financial point of view. Among the main economic aspects which can influence the network operation, we can point out price models, consumer behavior and the competition among cellular operators. In order to corroborate the advantages of a Radio Resource management based on economic aspects, we carry out a case study using computation simulation.

Keywords-Radio Resource Management

(RRM), Microeconomics, Consumer Behavior.

\section{INTRODUÇÃo}

A área de comunicações móveis é um dos segmentos que mais crescem atualmente. Isto ocorre tanto na esfera da indústria de insumos (aparelhos celulares, hardware de estações radio base e antenas) como na esfera das operadoras que fornecem o acesso sem fio aos consumidores. Apenas para exemplificar, no mês de março de 2007, 965.728 novos assinantes foram adicionados aos já existentes 101.186.709 acessos móveis em serviço no Brasil em fevereiro, resultando em um total de 102.152.437 assinantes do Serviço Móvel Pessoal (SMP) em todo o território brasileiro [1]. Este panorama é ainda esperado por muitos anos para a indústria, devido ao desenvolvimento e implantação das redes de terceira e futuras gerações, já que um acesso novo normalmente representa a venda de um aparelho novo. Entretanto, a recíproca não é verdadeira, ou seja, um novo aparelho vendido não significa um novo acesso contratado junto a alguma operadora. A aquisição de um novo terminal móvel pode perfeitamente ser troca do aparelho velho por um novo, mantendo o antigo acesso. Portanto, as operadoras se depararão com desafios mercadológicos num futuro próximo.

* Este estudante é apoiado por uma bolsa de estudo patrocinada pela FUNCAP. Os autores fazem parte do Grupo de Pesquisa em Telecomunicações Sem Fio - GTEL / Departamento de Teleinformática - DETI / Universidade Federal do Ceará - UFC. Emails: \{neto,rodrigo\}@gtel.ufc.br
De fato, nos dias atuais, as taxas de aumento da base de assinantes continuam a crescer, porém já é verificado tendências de queda nestas taxas de adesão de assinantes a nível mundial [2]. Como consequiência direta disto, os preços cobrados aos assinantes tendem a diminuir devido a lei da oferta e da procura. Se por um lado a saturação do número de assinantes é desafio para as operadoras, este fenômeno não assusta a indústria, pois é esperado que os consumidores já existentes provavelmente troquem seus aparelhos para se adaptarem a novos serviços e novas tecnologias.

Possivelmente, o efeito da saturação de assinantes poderá ser contrabalanceado pela receita gerada devido à exploração de novos serviços baseados em transmissão de dados. Estes novos serviços com características multimídia, disponibilizarão aos usuários acesso à Internet de banda larga. Além disso, deverão ser amplamente disponíveis, simples de contratar e fáceis de acessar, para um grande número de usuários. Para que todos estes pré-requisitos venham a ser satisfeitos é necessário não apenas empregar banda adicional, mas também realizar um gerenciamento eficiente e eficaz dos recursos de rádio da rede sem fio levando em consideração tanto as expectativas de desempenho como a disposição do usuário para pagar pelo serviço. Discutir como fazer este gerenciamento de recursos de rádio centrado no usuário é o assunto deste trabalho. Na seção II nós apontamos algumas direções de pesquisa que envolvem modelos de tarifação, modelagem do comportamento do consumidor de serviços de telecomunicações sem fio e estratégias de Gerenciamento de Recursos de Rádio (RRM, do inglês Radio Resource Management) do ponto de vista do usuário e competição entre operadoras. Na seção III, nós apresentamos um estudo de caso, onde é evidenciado através de resultados de simulação que um Gerenciamento de Recursos de Rádio centrado no comportamento do usuário pode trazer benefícios financeiros para a operadora. Finalmente, a seção IV apresenta algumas conclusões desta investigação.

\section{TÓPICOS DE INVESTIGAÇÃO}

Este estudo tem como objetivo investigar o Gerenciamento de Recursos de Rádio em redes de telefonia celular, abordando além dos quesitos técnicos tradicionais, quesitos econômicos. A análise destes quesitos econômicos, no contexto RRM, é um problema aberto pouco investigado na literatura técnica da área. A seguir seguem alguns tópicos de investigação.

\section{A. Modelos de Tarifação}

Quando se estuda o impacto econômico do Gerenciamento de Recursos de Rádio em redes sem fio, a estratégia de 
RRM adotada tem diferentes resultados de acordo com o modelo de tarifação utilizado [3], [4], [5]. Devido a isto é importante analisar as opções dos diversos modelos existentes e até mesmo propor novos modelos, que sejam justos tanto para os usuários como para as operadoras de acesso de rádio.

Na Figura 1 mostramos alguns exemplos de modelos já existentes na literatura e que serão estudados em conjunto com as técnicas de RRM [6]. O modelo 1(a) não leva em conta a quantidade de recursos oferecidos ao usuário no momento da tarifação, admitindo sempre um preço constante. O modelo 1(b) tarifa o usuário de maneira proporcional aos recursos oferecidos, sem levar em consideração os requerimentos do serviço utilizado. O modelo 1(c) protege o usuário de uma possível falta de qualidade, sendo a operadora, neste caso, obrigada a compensar o usuário por esta falta de qualidade, através de um preço negativo (bônus), a partir de uma certa quantidade de recurso mínima. O modelo 1(d) embute uma região de saturação, na qual não interessa mais ao usuário pagar por mais recursos. No entanto, a operadora pode oferecer recursos adicionais nos momentos de baixa carga da rede, visando uma desocupação mais rápida da rede e ao mesmo tempo uma maior satisfação (sem ônus) para os usuários.

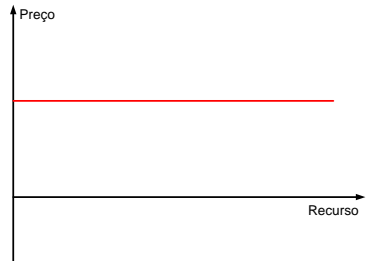

(a) Preço fixo.

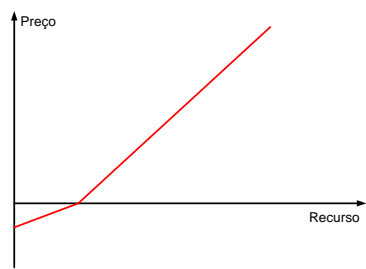

(c) Preço linear com penalidade.

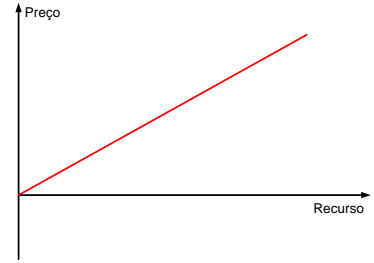

(b) Preço linear.

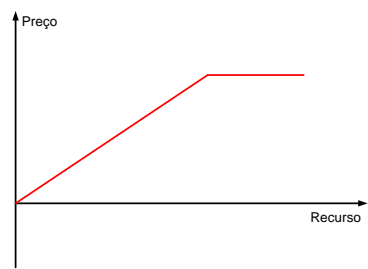

(d) Preço linear com saturação.
Fig. 1. Exemplos de modelos de tarifação.

\section{B. Modelagem do Comportamento do Consumidor}

Por se tratar de uma ciência, a economia está preocupada com a explicação e predição de fenômenos observados. Como em qualquer ciência, a explicação e predição são baseadas em teorias. Teorias são desenvolvidas para explicar fenômenos observados em termos de um conjunto de regras e hipóteses. Juntamente com técnicas econômicas e estatísticas, as teorias econômicas podem ser usadas para elaborar modelos, dos quais predições quantitativas podem ser feitas. Uma das áreas de pesquisa neste ramo é a modelagem do comportamento do consumidor (usuário, cliente) de serviços de telefonia móvel. Esta modelagem pode ser implementada utilizando alguns conceitos de Microeconomia como utilidade e probabilidade de aceitação do serviço.
1) Utilidade: Um dos conceitos da economia utilizados para modelar o comportamento do consumidor é a utilidade [7], [8], [9]. Utilidade é o nível de satisfação que uma pessoa obtém ao consumir um bem ou serviço. Este nível de satisfação pode ser mapeado através de uma função utilidade que tem como parâmetro a quantidade de bens consumidos e como saída um número real entre 0 e 1 (para o caso normalizado). No caso de redes sem fio, os bens consumidos são os recursos de rádio $r$, que podem estar relacionados a potência, taxa de transmissão, tempo de utilização de canal, entre outros. Então, neste caso, a satisfação do usuário está relacionada com a qualidade percebida pelo usuário ao utilizar o serviço sem fio.

Como as funções utilidades $u(r)$ mapeiam a qualidade percebida, elas são funções não decrescentes do parâmetro $r$, isto é, ela assume que um usuário não pode ficar menos satisfeito ao receber um acréscimo de recurso de rádio. Isto implica os seguintes requerimentos para as funções utilidades [3], [10]:

$$
\begin{gathered}
\frac{d u(r)}{d r} \geq 0, \\
\lim _{r \rightarrow \infty} \frac{d u(r)}{d r}=0 .
\end{gathered}
$$

A segunda condição (equação 2) é conhecida como o Princípio da Diminuição da Utilidade Marginal (medida da satisfação adicional obtida pelo consumo de uma quantidade adicional de um certo bem). Isto é consequiência do fato de que a partir de um certo ponto, o melhoramento da qualidade da conexão de rádio não é tão mais notável, mesmo que sejam utilizados mais recursos de rádio. Isto pode ser bem exemplificado através da métrica de qualidade conhecida como taxa de erro de bit (do inglês, BER), que é função da SINR (do inglês, Signal-to-Interference-plus-Noise Ratio). Quando a BER já está muito baixa, proporcionada por uma SINR muito alta, o aumento da SINR terá pouco impacto na diminuição da BER, pois esta já se encontra num nível imperceptível para o usuário. Como consequência do fenômeno da insensibilidade humana para o melhoramento da qualidade além de um certo limite, podemos escrever mais uma condição para as funções utilidades:

$$
\lim _{r \rightarrow \infty} u(r)=1
$$

Esta equação normaliza as funções utilidades, embora isto não seja um requerimento mandatório. Obviamente, o valor mínimo para a função utilidade é obtido quando é assinalado o valor mínimo de recurso, no caso $r=0$.

Como já foi dito, a utilidade tem um importante fator subjetivo, pois depende da percepção do usuário. A Figura 2 mostra diferentes tipos de funções utilidades que representam usuários com diferentes requisitos de qualidade. A primeira delas é apropriada para um usuário de um serviço de "melhor esforço"(isto é, não requer restrição forte no atraso de entrega da informação, por exemplo, acesso a uma página WWW da Internet), enquanto a segunda é adequada para um usuário de serviço de tempo real (ou seja, serviço que requer atraso máximo de entrega garantido). No primeiro caso, a queda na 
satisfação do usuário é suave devido à restrição de atraso ser fraca, enquanto no segundo caso, a partir de um certo ponto na quantidade de recursos oferecidos, a queda na satisfação é abrupta e tende para zero devido à forte restrição de atraso. Portanto é necessário investigar estratégias para a construção de funções utilidades mais fidedignas quanto possível, aplicáveis ao segmento de telecomunicações sem fio, seja através da otimização de parâmetros bem como através de outros procedimentos probabilísticos e matemáticos. Outro tópico de pesquisa ainda não explorado é a elaboração de funções utilidades multidimensionais, onde a alocação de dois ou mais tipos diferentes de recursos de rádio determinará a satisfação dos usuários.

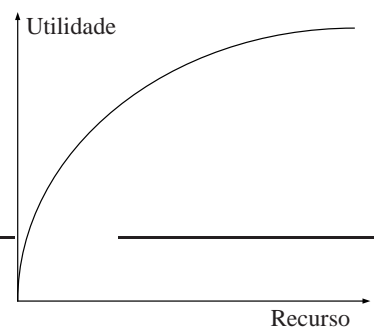

(a) Tráfego do tipo "melhor esforço".

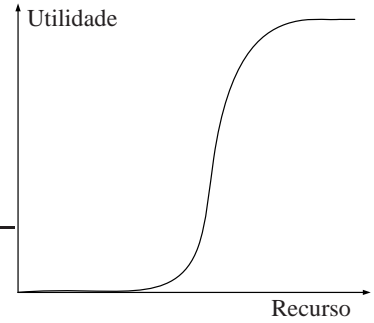

(b) Tráfego de tempo real.
Fig. 2. Utilidades para tráfego do tipo "melhor esforço"e tempo real.

2) Probabilidade de Aceitação do Serviço: Outro conceito que descreve o comportamento do usuário é a probabilidade de aceitação do serviço. A probabilidade de aceitação de um determinado serviço depende principalmente de dois fatores: o preço cobrado pelo serviço e a utilidade obtida pelo serviço. Quanto maior for o preço a ser pago pelo serviço, menos o usuário estará disposto a aceitá-lo. Por outro lado, quanto maior for a qualidade do serviço oferecido, o usuário estará mais disposto a aceitá-lo.

Como foi visto na seção II-A, o preço é função da quantidade de recursos alocados. A utilidade, por sua vez, também é função da quantidade de recursos alocados. Portanto a probabilidade de aceitação é uma função da quantidade de recursos alocados. Então, vale a pena enfatizar que a quantidade de recursos pode influenciar a probabilidade de aceitação de duas maneiras inversas: quanto maior a quantidade de recursos, maior o preço cobrado, o que diminui a aceitação, porém maior será a utilidade do usuário, o que aumenta a probabilidade de aceitação.

A Microeconomia já estuda este comportamento do consumidor através dessas funções. De acordo com seu significado e objetivo, uma função de probabilidade de aceitação $A$ devem satisfazer as seguintes propriedades [3]:

$$
\begin{gathered}
\frac{\partial A}{\partial u} \geq 0, \quad \frac{\partial A}{\partial p} \leq 0 . \\
\forall p>0, \quad \lim _{u \rightarrow 0} A(u, p)=0, \quad \lim _{u \rightarrow \infty} A(u, p)=1 .
\end{gathered}
$$

$$
\forall u>0, \quad \lim _{p \rightarrow 0} A(u, p)=1, \quad \lim _{p \rightarrow \infty} A(u, p)=0 .
$$

Uma função bastante utilizada em Microeconomia e que satisfaz estas condições são as curvas de demanda Cobb-Douglas [7], [8], [9] que podem ser expressas como:

$$
A(u, p)=1-e^{-C u^{\mu} p^{-\varepsilon}},
$$

onde $\mathrm{C}, \mu \mathrm{e} \varepsilon$ são constantes positivas que refletem o perfil do usuário. A constante $\mu$ indica a sensibilidade do usuário em relação a utilidade, enquanto $\varepsilon$ indica a sensibilidade em relação ao preço.

Estudos adicionais a respeito da aceitação do consumidor de serviços de telecomunicação sem fio necessitam ser explorados e aprofundados, pois estas funções de probabilidade de aceitação servem como subsídios tanto para a fase de planejamento da rede como para a fase de operação.

\section{Estratégias de Gerenciamento de Recursos de Rádio}

Vimos que a quantidade de recursos alocados $r$ para um usuário determina o preço $p$, a utilidade $u$ e a probabilidade de aceitação $A$ do serviço. O Gerenciamento de Recursos de Rádio é o responsável por esta alocação, portanto a entidade relacionada a esta função tem um papel fundamental nestes três conceitos microeconômicos e vice-versa. Então, é desejável pesquisar técnicas inovadoras que levam em consideração este impacto econômico.

Considerando o recurso alocado como a taxa de transmissão, podemos identificar algumas métricas estatísticas que podem servir como função objetivo para o Gerenciamento de Recursos de Rádio, tais como a receita gerada $R$, o número de usuários $S$ que aceitam o comprar o serviço, a quantidade de recursos alocados $T$, e a utilidade total $U$ dos usuários admitidos [11], [12]:

$$
\begin{gathered}
R=\sum_{i=1}^{N} p_{i} A\left(u_{i}, p_{i}\right) . \\
S=\sum_{i=1}^{N} A\left(u_{i}, p_{i}\right) . \\
T=\sum_{i=1}^{N} r_{i} A\left(u_{i}, p_{i}\right) . \\
U=\sum_{i=1}^{N} u_{i} A\left(u_{i}, p_{i}\right) .
\end{gathered}
$$

onde $N$ é a quantidade de usuários do sistema.

Ou seja, nós podemos escolher diferentes metas de RRM, ou até mesmo propor um arcabouço de métricas conjuntas, porém de prazos diferentes (maximização de longo prazo e curto prazo), já que algumas métricas requerem decisões contraditórias. Por exemplo: maximizar em curto prazo a receita e a longo prazo a utilidade total. 


\section{Competição entre Operadoras de Acesso de Rádio}

Num cenário onde é dado ao usuário a possibilidade de escolha da operadora, no momento da aquisição do serviço de telecomunicação, a estratégia de RRM adquire um papel fundamental na concorrência entre as diversas operadoras.

Isto ocorre porque os recursos alocados impactam diretamente na probabilidade de aceitação do usuário. Então, para a operadora, é importante elaborar uma estratégia de alocação de recursos que estimule o usuário a adquirir seus serviços e ao mesmo tempo preserve sua receita e sua capacidade de suportar novos usuários.

Como a alocação de recursos influencia a probabilidade de aceitação e esta, por sua vez, influencia na escolha feita pelo usuário a respeito da operadora, é facilmente dedutível que a estratégia de alocação de recursos impactará na concorrência comercial entre as operadoras. Adicionalmente, o modelo de tarifação também será um fator importante nesta concorrência.

Portanto é necessário planejar conjuntamente tanto a estratégia de RRM como a política de preços, visando um desempenho máximo no ambiente comercial competitivo. Uma das ferramentas adequadas para solucionar este problema é Teoria dos Jogos.

A Teoria dos Jogos é uma ferramenta matemática que estuda a interação entre agentes (jogadores) [13]. Ela é aplicável quando a ação de um agente tem impacto sobre o desempenho dos demais, que é o caso da competição comercial. O objetivo da Teoria dos Jogos é o fornecimento de subsídios para a tomada de decisões e a previsão do resultado de suas ações. Portanto, acreditamos que o emprego de Teoria dos Jogos na concorrência do setor de telecomunicações, tendo como possíveis ações dos jogadores (neste caso as operadoras), as escolhas da estratégia de RRM e do modelo de tarifação, será de grande valor prático e acadêmico, já que na literatura este estudo é muito raro.

Outra ferramenta matemática adequada a esta investigação é a Otimização Estocástica, útil em problemas que tentam modelar a incerteza em dados de entrada, assumindo que estes (ou parte deles) são especificados em termos de uma distribuição de probabilidade [14]. Em nosso caso, a incerteza no processo de otimização estará presente na demanda dos usuários pelos serviços wireless. Então acreditamos no potencial desta técnica para a solução do problema proposto.

\section{Estudo de CAso: MinimizaÇÃo DA TAXA DE Churn}

Para evidenciar os possíveis ganhos de se realizar um Gerenciamento de Recursos de Rádio baseado em modelos econômicos, nós investigamos um cenário no qual uma operadora de telefonia celular tenta maximizar a permanência de seus usuários após um determinado período de utilização de seus serviços. Durante este determinado período, vários usuários fazem diversas chamadas e acumulam um histórico de satisfação. Se após este período algum usuário estiver insatisfeito com a média histórica da qualidade de suas chamadas, ele desistirá de continuar nesta operadora e provavelmente migrará para outra operadora. Tal fenômeno é denominado churning. Portanto, o objetivo da operadora é reduzir sua taxa de churn, que é a porcentagem de desistência em sua base de assinantes na continuação do consumo dos serviços prestados por ela. Assumimos que a operadora escolhe sua estratégia de alocação de recursos no início do período de investigação e esta estratégia se mantêm inalterada durante este período.

\section{A. Modelo do Sistema}

Nós usamos um modelo de sistema que representa o acesso de um único canal compartilhado por um conjunto de usuários para transmissão de dados, em um ambiente unicelular. Nossa modelagem inclui um modelo completo de tráfego, uma interface aérea genérica bem como a funcionalidade de RRM representada pelo escalonamento temporal de usuários. Quando um usuário é escalonado, a ele é dado toda a potência da Estação Radio Base. Assim, quando o usuário está transmitindo, ele não sofre interferência. As fontes de degradação do sistema são a perda de percurso, sombreamento, ruído (que impactam diretamente na SNR dos usuários) e a própria competição pelo canal, que deve ser gerenciada pela entidade de RRM. Mobilidade real não é considerada, mas uma variação periódica do sombreamento é realizada, visando dar um caráter mais realista às simulações.

A taxa de chegada de cada usuário é modelada como um processo de Poisson com uma taxa de chegada específica $\lambda$. Apenas um tipo de serviço do tipo "melhor esforço"será estudado neste caso. A modelagem do tráfego é feita a nível de sessão. Para isto, nós usamos um modelo de download, onde o tamanho da sessão é dado por uma função densidade de probabilidade log-normal de média 2,8 Mbytes. Devido a característica deste serviço do tipo "melhor esforço", não incluímos bloqueio nem "dropping”de usuário.

A taxa de transmissão $R$ do usuário é dada pela curva de adaptação de enlace. Com propósitos de simplificação, nós usamos uma curva de mapeamento linear idealizada, mostrada na figura 3. As variáveis que caracterizam esta curva idealizada são a SNR de saturação máxima, $S N R_{\max }$ e a SNR mínima, $S N R_{\text {min }}$, bem como suas respectivas taxas de transmissão, $R_{\max }$ e $R_{\text {min }}$.

O principal indicador de desempenho de nossa simulação é a taxa de bit CSE (Circuit Switched Equivalent) que é determinada baseada em conceitos de teoria das filas [15]. Esta taxa mede a velocidade em que os buffers dos usuários podem ser esvaziados e, portanto, inclui efeitos de enfileiramento.

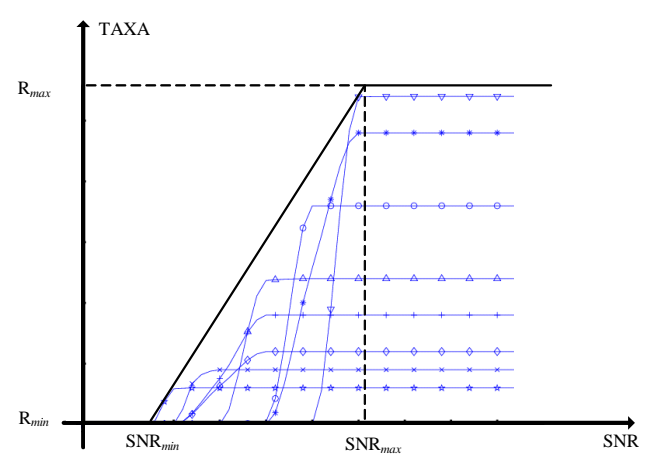

Fig. 3. Curva de adaptação de enlace. 
Portanto ela está diretamente relacionada com a qualidade da transmissão percebida pelo usuário.

O CSE de cada usuário é calculado a partir do fator efetivo de utilização de link $\rho$ dos usuários. Esta grandeza mede a fração do tempo entre dois eventos consecutivos no qual o canal de transmissão é alocado para o usuário. É responsabilidade da entidade de RRM definir a melhor estratégia de divisão do canal, ou seja, determinar o fator $\rho$ de cada usuário.

De posse dos fatores efetivos de utilização de link de todos os usuários e da taxa de transmissão do usuário $i$, a taxa de bit CSE do usuário $i$ é calculada como:

$$
C S E_{i}=R_{i} \cdot\left(1-\sum_{j \neq i} \rho_{j}\right) .
$$

O que define a duração de cada chamada, são a quantidade de dados que o usuário tem para transmitir, a taxa de transmissão $R$ e o tempo efetivo de canal que é dado ao usuário que depende do fator efetivo de utilização de link, que por sua vez, é definido pela estratégia de RRM. Ao final de cada chamada de cada usuário é calculada e armazenada a satisfação deste usuário nesta chamada que é dada pela utilidade calculada da seguinte maneira:

$$
u_{i}\left(\overline{C S E}_{i}\right)=\frac{\left(\overline{C S E}_{i} / \beta_{i}\right)^{\alpha_{i}}}{1+\left(\overline{C S E}_{i} / \beta_{i}\right)^{\alpha_{i}}},
$$

onde $\overline{C S E}_{i}$ é a taxa CSE média do usuário $i$ durante a chamada, $\alpha_{i}$ e $\beta_{i}$ são parâmetros de ajuste usados para diferenciar os usuários entre si e depende da classe de serviço do usuário. Note que esta função utilidade é normalizada, de modo que ela é limitada entre 0 e 1 . Além disso ela satisfaz as propriedades assintóticas e obedece à lei do decréscimo marginal, propriedade comum a grande maioria de funções utilidade.

Procedendo desta maneira, após o período de investigação $T$, cada usuário $i$ da base de assinantes terá vários valores de utilidade, uma para cada chamada. Com estes valores, calculamos sua utilidade média $u_{i}^{T}$ do período $T$.

De posse da utilidade média $u_{i}^{T}$ e do preço $p$ praticado durante o período $T$ pela operadora, calculamos para cada usuário uma probabilidade de continuidade de operadora. A probabilidade de continuidade segue a forma da probabilidade de aceitação previamente apresentada na subseção II-B.2:

$$
P_{i}\left(u_{i}^{T}, p\right)=1-e^{-C \cdot\left(u_{i}^{T}\right)^{\mu} \cdot p^{-\varepsilon}},
$$

onde $\mathrm{C}, \mu \mathrm{e} \varepsilon$ são constantes positivas que refletem o perfil do usuário. A constante $\mu$ indica a sensibilidade do usuário em relação a utilidade, enquanto $\varepsilon$ indica a sensibilidade em relação ao preço.

Obviamente, o modelo de tarifação também influencia a taxa de churn. No entanto, como estamos interessados apenas no efeito da alocação de recursos neste estudo de caso, consideramos o modelo flat (Figura 1(a)) nas simulações computacionais realizadas.

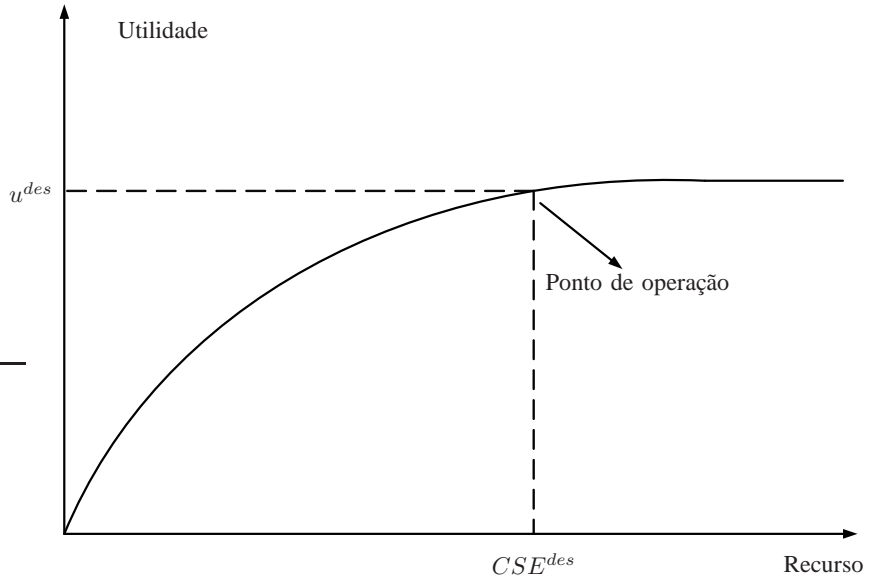

Fig. 4. Algoritmo baseado em utilidade.

\section{B. Algoritmos Avaliados}

Para verificarmos o efeito da alocação de recursos, comparamos dois algoritmos de escalonamento. O primeiro é o algoritmo clássico Round Robin em que não são levados em consideração os aspectos econômicos. O segundo é um algoritmo proposto baseado em utilidade. Este último possui uma reserva de recursos (neste caso 10\%), que é alocada para o usuário de pior histórico de utilidade até o momento. Para os recursos não reservados (neste caso 90\%), os usuários compartilham o canal de acordo com o conceito de fator desejado de utilização de link. $O$ fator desejado de utilização de link do usuário $i$ é definido como:

$$
\rho_{i}^{d e s}=\frac{C S E_{i}^{d e s}}{R_{i}},
$$

onde $C S E_{i}^{\text {des }}$ e $R_{i}$ são a taxa de bit CSE desejada e a taxa de transmissão (dada pela curva de adaptação de enlace) do usuário $i$, respectivamente. Uma vez que esses fatores são calculados para cada usuário, o escalonador vai alocando recursos para os usuários que possuem os menores $\rho_{i}^{d e s}$, até que os recursos se esgotem. Desse modo, usuários com a maior taxa de transmissão $R_{i}$, terão prioridade na alocação de recursos.

Então, esta estratégia, ao mesmo tempo em que tenta elevar a utilidade média dos usuários de pior histórico de utilidade, aloca recursos para os usuários de melhor taxa numa quantidade suficiente para que naquele momento ele atinja uma elevada utilidade sem desperdiçar recursos que subjetivamente não alteraria a satisfação do usuário de maneira significante. A Figura 4 ilustra o princípio de funcionamento do esquema proposto.

\section{Resultados de Simulação}

Neste estudo de caso, admitiremos que todos os usuários têm as mesmas características de satisfação, portanto, as constantes $\alpha_{i}$ e $\beta_{i}$ são as mesmas para todos os usuários e emulam um comportamento do usuário característico de serviço de "melhor esforço", similar a Figura 2(a). Simulamos 3 diferentes tempos entre chamadas do processo de Poisson no sistema: 20, 22 e 24 segundos. A base de assinantes é de 


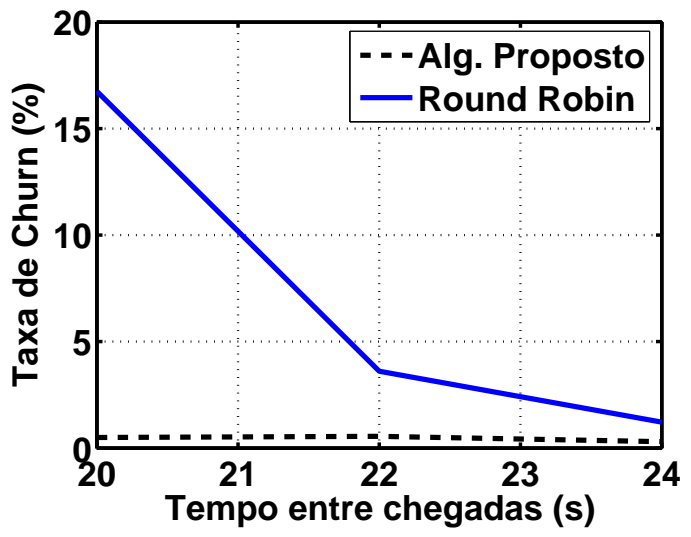

Fig. 5. Taxa de churn para diferentes cargas no sistema.

360 usuários e o período de observação $T$ corresponde a 2 meses. Para o algoritmo proposto, adotamos um mesmo valor de $C S E^{\text {des }}$ para todos os usuários igual a $512 \mathrm{Kbps}$, o que corresponde a uma utilidade desejada de 0,995 . Os demais parâmetros de simulação são dados na Tabela I.

Pode-se observar que o algoritmo proposto apresenta melhor desempenho que o algoritmo Round Robin para todas as cargas simuladas. Para a maior carga, enquanto o algoritmo proposto resultou numa taxa de churn de $0,5 \%$, o Round Robin atinge uma taxa de churn de 16,75\%. Pode-se ver também que o esquema proposto parece dar uma maior robustez ao sistema, visto que com o aumento da carga não há grande variação na taxa de churn do sistema, ao contrário do acontece com o Round Robin.

Portanto, estes resultados de simulação sugerem que um Gerenciamento de Recursos de Rádio centrado no comportamento do usuário pode trazer benefícios financeiros para a operadora, pois a redução na taxa de churn da operadora acarretará diretamente numa diminuição da perda de receita devido a migração de usuários para uma operadora concorrente.

TABELA I

PARÂMETRos DE SimulaÇÃo.

\begin{tabular}{c|c}
\hline \hline Parâmetro & Valor \\
\hline \hline$S N R_{\min }$ & $-17 \mathrm{~dB}$ \\
\hline$S N R_{\max }$ & $7 \mathrm{~dB}$ \\
\hline$R_{\min }$ & $64 \mathrm{kbps}$ \\
\hline$R_{\max }$ & $3,6 \mathrm{Mbps}$ \\
\hline Raio da célula & $0,5 \mathrm{Km}$ \\
\hline Expoente de Propagação & 4 \\
\hline Desvio padrão do sombreamento & $6 \mathrm{~dB}$ \\
\hline Potência de transmissão & $43 \mathrm{dBm}$ \\
\hline Potência do ruído & $-110 \mathrm{dBm}$ \\
\hline$C$ & 0,0458 \\
\hline$\mu$ & 2 \\
\hline$\beta$ & 4 \\
\hline$\alpha$ & 224320 \\
\hline Período de atualização do sombreamento & 6,4142 \\
\hline \hline
\end{tabular}

\section{Conclusĩo}

Este trabalho contribuirá para o aperfeiçoamento do gerenciamento de recursos de rádio no que diz respeito à incorporação de aspectos econômicos aos aspectos técnicos já utilizados. O desenvolvimento do novo arcabouço em termos da alocação de recursos de uma maneira geral permite uma ampla utilização da técnica desenvolvida, possibilitando o seu emprego em vários ramos do Gerenciamento de Recursos de Rádio como: alocação de potência, alocação de taxa, escalonamento, controle de admissão e outros. Acreditamos que o emprego de ferramentas econômicas tem bastante espaço para inovação e portanto será de grande valia e relevância para a pesquisa em telecomunicações.

\section{REFERÊNCIAS}

[1] Release Anatel 01/05/2007. Online. Disponível em: http://www.anatel.gov.

[2] P. Nicopolitidis, G. Papadimitriou, M. S. Obaidat e A. S. Pomportsis, "The Economics of Wireless Networking", Communications of the ACM, vol. 47, No 4, pp. 83-86, Abril 2004.

[3] L. Badia, M. Lindstrom, J. Zander e M. Zorzi, "An Economic Model for the Radio Resource Management in Multimedia Wireless Systems", Globecom, pp. 4116-4121, 2003.

[4] L. Badia, M. Lindstrom, J. Zander e M. Zorzi, "Demand and Pricing Effects on the Radio Resource Allocation of Multimedia Communication Systems", Computer Communications, No 27, pp. 1056-1064, 2004.

[5] L. Badia e M. Zorzi, "An Analysis of Multimedia Services in Next Generation Communication Systems with QoS and Revenue Management", Vehicular Technology Conference - Spring, pp. 2012 2016, Maio 2004

[6] N. C. Ericsson, "Revenue Maximization in Resource Allocation: Applications in Wireless Communication Networks", Tese de Doutorado, Uppsala Universitet, October 2004.

[7] R. S. Pindyck e D. L. Rubinfeld, "Microeconomics", Prentice-Hall, $3^{\text {nd }}$ edition, 1996.

[8] H. R. Varian, "Microeconomia, Princípios Básicos", Editora Campus, $6^{\mathrm{a}}$ edição, 2003.

[9] F. A. Cowell, "Microeconomics: Principles and Analysis", Dezembro de 2004.

[10] M. Lindstrom, L. Badia, J. Zander e M. Zorzi, "Speculative Resource Allocation for Packet-Switched Wireless Networks", Vehicular Technology Conference-Spring, 2005.

[11] L. Badia e M. Zorzi, "On Utility-based Radio Resource Management with and without Service Guarantees", MSWiM, 2004.

[12] L. Badia, C. Saturni, L. Brunetta e M. Zorzi, "An Optimization Framework for Radio Resource Management Based on Utility vs. Price Tradeoff in WCDMA Systems", Third International Symposium on Modeling and Optimization in Mobile, Ad Hoc, and Wireless Networks, 2005.

[13] T. Basar e G. J. Olsder, "Dynamic Noncooperative Game Theory", Academic Press, $2^{\text {nd }}$ edition, 1995.

[14] M. G. C. Resende and P. M. Pardalos, "Handbook of Optimization in Telecommunications", Springer, 2006.

[15] A. Furuskar, "Can 3G Services Be Offered in Existing Spectrum ?", Licentiate Thesis, Royal Institute of Technologie, Stockholm, Sweden, May 2001. 